\title{
Differential Maturation of the Innate Immune Response in Human Fetuses
}

\author{
TOBIAS STRUNK, PETRA TEMMING, ULRICH GEMBRUCH, IRWIN REISS, \\ PETER BUCSKY, AND CHRISTIAN SCHULTZ
}

\author{
Department of Pediatrics [T.S., P.T., P.B., C.S.], University of Lübeck, Medical School, 23538 Lübeck, \\ Germany; Department of Obstetrics and Prenatal Medicine [U.G.], University of Bonn, 53105 Bonn, \\ Germany; and Department of Pediatrics [I.R.], Justus Liebig-University Giessen, 35392 Giessen, Germany
}

\begin{abstract}
Newborns and especially preterm infants show a unique susceptibility to severe bacterial infections that cause significant morbidity and mortality. As very few data are available on innate immune functions in human fetuses, we conducted a comprehensive study to investigate the expression of several adhesion molecules essentially involved in migration (CD11a, CD11b, CD11c, CD18, and CD62L). Furthermore, phagocytic activity, generation of respiratory burst products, and production of several proinflammatory cytokines were assessed. Various functions of the fetal innate immune system were demonstrated to be essentially different from those observed in term neonates or adults. Expression of several surface markers was significantly diminished on fetal granulocytes. Furthermore, a significantly reduced phagocytic activity of fetal granulocytes and monocytes was found, contrasted by an enhanced generation of reactive oxygen products. In addition, we demonstrate that significant
\end{abstract}

\section{ABSTRACT}

numbers of fetal monocytes are capable of the production of proinflammatory cytokines in response to stimulation. However, the pattern of cytokine production is different from the more mature individuals: the number of IL-6- and tumor necrosis factor- $\alpha$-positive monocytes were significantly diminished, whereas more IL-8-producing monocytes were found compared with adults. The results of our study add significantly to our understanding of the maturation and impairment of the innate immune response. (Pediatr Res 56: 219-226, 2004)

Abbreviations
fMLP, N-formyl-methionyl-leucyl-phenylalanine
MFI, mean fluorescence intensity
NRBA, neutrophil respiratory burst activity
TNF- $\alpha$, tumor necrosis factor- $\alpha$

Neonates, especially preterm infants, are prone to severe and overwhelming bacterial infection with substantial morbidity and mortality. Despite intensive supportive care and early use of antibiotics, the rate of morbidity and mortality caused by infections remains high (1). Although multiple factors contribute to this susceptibility, the immaturity of the innate immune system, especially production of phagocytes and several phagocyte functions (e.g. chemotaxis, phagocytosis, respiratory burst, cytokine production) are of paramount importance $(2,3)$. Evidence of this is provided by the pattern of infections closely resembling that seen in profound neutropenia (4). Depleted neutrophil storage pools and neutropenia were described to be associated with neonatal sepsis and are negative predictors of outcome $(5,6)$. Several qualitative deficiencies of phagocyte function were demonstrated earlier in preterm in-

Received July 15, 2003; accepted March 16, 2004.

Correspondence: Tobias Strunk, M.D., Department of Pediatrics, University of Lübeck, Medical School, Ratzeburger Allee 160, 23538 Lübeck, Germany; e-mail: strunk@ paedia.ukl.mu-luebeck.de or tobiasstrunk@yahoo.de

This study was supported in part by the "Friedrich Bluhme and Else Jebsen StiftungLübeck" and Research Grant of the University of Lübeck, Grant No. 3601.

DOI: 10.1203/01.PDR.0000132664.66975.79 fants and stressed or septic neonates. The functional deficiencies comprised migration, phagocytosis, and bactericidal potency, for example, release of bactericidal/permeabilityincreasing protein (7-15). However, normal or even enhanced spontaneous neutrophil respiratory burst activity in term and preterm neonates was reported in other studies $(16,17)$. In contrast, little is known to date about the phagocytic and oxidative capacity as well as the adhesion molecule expression of fetal neutrophils and monocytes. The expression of several adhesion molecules essentially involved in migration was reported to be significantly impaired in neonates and to contribute to the impaired neonatal immune response (18-20); however, only very limited data are available for fetuses (21). Similarly, to our knowledge, there are no reports on the ability of fetal monocytes to produce proinflammatory cytokines in response to endotoxin challenge. To further elucidate these intriguing questions, we investigated the expression of several migration-associated adhesion molecules, phagocytosis, and respiratory burst activity as well as synthesis of proinflammatory cytokines at the single-cell level in cord blood of human fetuses obtained by percutaneous umbilical vein sampling in 
comparison with healthy neonates and adults. Analysis of these essential functions of the innate immune response significantly contributes to our understanding of the developing host defense.

\section{METHODS}

Blood samples and study population. The study was approved by the local institutional review board. Blood samples were taken after informed consent from healthy adults $(n=15)$ and from cord blood of healthy term neonates $(n=20$; mean weight, $3319 \mathrm{~g}$; range, 2650-4160 g). Blood samples from human fetuses [phagocytosis, oxidative burst, and adhesion molecule expression: $n=14$; mean gestational age, $28.4 \mathrm{wk}$ (range, 21.1-32.4 wk); intracellular cytokine analysis: $n=12$; mean gestational age, $26.0 \mathrm{wk}$ (range, 21.6-35.0 wk)] were taken by sonographically guided percutaneous umbilical vein sampling when medically indicated after informed consent was obtained. For ethical reasons, it is not possible to do elective cord blood sampling in normal, healthy fetuses. Therefore, we had to restrict the study to fetuses with medical indications for cord blood sampling, i.e. with abnormalities detected by routine investigations during pregnancy. Medical indications were diagnostic (karyotype analysis, fetal $\mathrm{Hb}$ level) as well as therapeutic (fetal transfusion). Fetuses in the first group (analysis of phagocytosis, oxidative burst, and adhesion molecule expression) had diagnoses of blood group incompatibility, hydrothorax, cardiac anomaly, lymph hemangioma, oligohydramnios, polyhydramnios, renal anomaly, situs ambiguous, and uteroplacental dysfunction. With the exception of two (trisomy 13 and 18), all karyotype analyses revealed normal results. Fetuses in the second group (intracellular cytokine analysis) had diagnoses of alloimmune-thrombocytopenia, blood group incompatibility, renal anomaly, intrauterine growth retardation, hydrocephalus, osteogenesis imperfecta, and diaphragmatic hernia. With the exception of one (trisomy 21), all karyotype analyses revealed normal results. Because of a small fetal sample size, experiments were done sequentially on the indicated number of individuals. Not all measurements were completed for each fetus because of occasional inadequate sample size. The figure legends give the number of fetuses analyzed in each specific data set.

Quantification of oxidative burst products and phagocytosis activity. Three-color flow cytometric analysis was performed on an EPICS XL flow cytometer (Coulter Electronics, Krefeld, Germany). All reagents, including preopsonized Escherichia coli, were supplied as stock solutions in Bursttest and Phagotest kits from Orpegen (Heidelberg, Germany), and procedures were done according to the manufacturer's recommendations. Briefly, aliquots of heparinized whole blood were preincubated for $10 \mathrm{~min}$ on ice to equilibrate starting conditions. After that, leukocytes were stimulated either by $10 \mu \mathrm{L}$ of N-formyl-methionyl-leucyl-phenylalanine (fMLP) solution $10^{-6} \mathrm{M}$ or by opsonized $E$. coli bacteria that were adjusted to a bacteria/leukocyte ratio of $25: 1$, and cells were incubated for $10 \mathrm{~min}$ at $37^{\circ} \mathrm{C}$. Oxidative burst products were quantified by the addition of $20 \mu \mathrm{L}$ of dihydrorhodamine 123 for $10 \mathrm{~min}$ at $37^{\circ} \mathrm{C}$, which was oxidized to the brightly fluorescent rhoda- mine 123 (dihydrorhodamine). The reaction was stopped by fixation of leukocytes and by lysis of erythrocytes with $2 \mathrm{~mL}$ of lysis buffer. After one washing step, the propidium iodide solution was added $15 \mathrm{~min}$ before measurement for counterstaining of the leukocyte nuclei. The amount of phagocytosed bacteria was measured by the fluorescence of FITC-labeled $E$. coli expressed as mean fluorescence intensity (MFI). Aliquots of heparinized whole blood were cooled at $0^{\circ} \mathrm{C}$ for $15 \mathrm{~min}$ and then incubated with a saturating concentration of $E$. coli for 10 min at $37^{\circ} \mathrm{C}$. A total of $100 \mu \mathrm{L}$ of cold quenching solution was added both to stop the reaction and to quench the fluorescence of bacteria adherent to the cell membrane. After two washing steps, the erythrocytes were lysed in $2 \mathrm{~mL}$ of lysing solution for $20 \mathrm{~min}$ at room temperature. After washing of the cells, 100 $\mu \mathrm{L}$ of phycoerythrin DNA stain was added and the cells were incubated at $0^{\circ} \mathrm{C} 10 \mathrm{~min}$ before analysis. PBS and incubation at $0^{\circ} \mathrm{C}$ during the whole procedure served as negative controls in the oxidative burst and phagocytosis assay, respectively.

The data were analyzed with Coulter EPICS XL System II, Version 1.0 software. Granulocytes and monocytes were gated according to their forward and side scatter characteristics. At least 2000 granulocytes and 1000 monocytes were analyzed, and data were expressed as a percentage of neutrophils and monocytes producing oxygen metabolites and phagocytosing E. coli as well as the MFI of the cells.

Cell surface marker staining. Granulocytes were stained with fluorescence-conjugated MAbs against CD11a, CD11b, CD11c, CD18, and CD62L (Coulter). Erythrocytes were lysed using Immuno-Lyse solution (Coulter). Background staining was carried out by using an unrelated fluorescein-labeled mouse antibody of the same isotype. A minimum of 5000 cells of each sample were analyzed.

Intracytoplasmic detection of cytokines. Flow cytometric analysis of cytokine production was done as described earlier $(22,23)$. Briefly, heparinized whole blood was suspended in RPMI 1640 supplemented with $1 \%$ penicillin/streptomycin, 2 $\mathrm{mM}$ of glutamine, $1 \mathrm{mM}$ of pyruvate, and nonessential amino acids at a concentration of $2 \times 10^{6}$ leukocytes $/ \mathrm{mL}$. Leukocytes were stimulated for $5 \mathrm{~h}$ in multiwell plates with $30 \mathrm{ng} / \mathrm{mL}$ of lipopolysaccharide (Sigma Chemical Co., Deisenhofen, Germany) at $37^{\circ} \mathrm{C}$ and $5 \% \mathrm{CO}_{2}$. All cultured cells were incubated in the presence of $3 \mu \mathrm{M}$ of monensin (Sigma Chemical Co.) to inhibit cytokine export. After a washing step with Hanks' balanced salt solution (Sigma Chemical Co.), cultured cells were fixed in 4\% paraformaldehyde (Riedel de Haen, Seelze, Germany) for $10 \mathrm{~min}$ and resuspended in nonfat dry milk (5\%) for $16 \mathrm{~h}$ at $4^{\circ} \mathrm{C}$ in the dark. Cells were permeabilized twice in a buffer consisting of Hanks' balanced salt solution (Seromed Biochrome, Berlin, Germany), 0.1\% saponin (Riedel de Haen), and 0.01 M of HEPES. Subsequently, cells were incubated for $20 \mathrm{~min}$ at $4^{\circ} \mathrm{C}$ in the dark with MAbs against CD14, IL-6, IL-8, and tumor necrosis factor- $\alpha$ (TNF- $\alpha$; Pharmingen, Heidelberg, Germany). Preincubation with an excess of unlabeled anticytokine MAbs for $20 \mathrm{~min}$ at $4^{\circ} \mathrm{C}$ in the dark served as a negative control for intracellular staining to each sample (21). Monocytes were gated by means of their CD14 positivity, and number of cytokine-positive cells as well as the MFI were measured of a minimum of 2000 cells per sample. 
Statistical analysis. Statistical differences among all groups were first checked by the Kruskal-Wallis test. After confirmation of significance, statistical differences were tested by the nonparametric Mann-Whitney $U$ test. The level of significance was defined as $P<0.05$ in single comparisons. It was corrected according to Bonferroni in multiple comparisons among groups $(\alpha=0.05 / 2=0.025)$. Correlation analysis was done by Spearman's rank-order test. Data are expressed as median and 25th to 75th percentiles, if not indicated otherwise. Statistical analyses were performed using SPSS 9.0 statistical software (SPSS Inc., Chicago, IL, U.S.A.).

\section{RESULTS}

Phagocytosis of E. coli bacteria in fetal versus neonatal and adult granulocytes and monocytes. Fetuses demonstrated significantly lower number of $E$. coli-phagocytosing granulocytes (median $75.2 \%$, 25th-75th percentile $49.2-80.3 \%$ versus $95 \%, 91.2-98.6 \% ; P<0.001)$ and smaller number of ingested bacteria as measured by MFI than healthy adults (MFI: 14.2, 11.5-29.4 versus $95.1,52.2-138.2 ; P<0.001)$. Similarly, the number of monocytes positive for bacteria $(60 \%, 48.4-74.1 \%$ versus $77.1 \%, 67.4-82.4 \% ; P=0.018)$ as well as the number of ingested bacteria was significantly lower in fetuses compared with healthy adults (MFI: 12.9, 7.8-19.8 versus 55.4, 41.6-68.2, $P<0.001$; Fig. 1). A direct positive correlation between fetal gestational age and number of $E$. coli-positive granulocytes was observed ( $r=0.73, P<0.05)$.

Compared with healthy neonates, fetuses displayed significantly diminished number of bacteria ingested by granulocytes
(MFI: 14.2, 11.5-29.4 versus 48.7, 33.9-69.2, $P<0.001$ ); phagocytosing granulocytes also showed a trend toward lower frequency $(75.3 \%, 49.2-80.3 \%$ versus $86.0 \%, 76.5-89.2 \% P$ $=0.03$ ). There was no statistically significant difference in the number of phagocytosing monocytes between fetuses and neonates $(60 \%, 48.4-74.1 \%$ versus $71.6 \%, 60.8-75.9 \% ; P=$ 0.3 ; Fig. 1); however, number of ingested bacteria was significantly lower in fetal versus neonatal monocytes (MFI: 12.9, 7.8-19.8 versus $34.1,25.3-45.1 ; P<0.001)$.

Healthy neonates showed decreased phagocytotic capacity in terms of granulocytes positive for bacteria $(86.0 \%, 76.5-$ $89.2 \%$ versus $95 \%, 91.2-98.6 \% ; P<0.001)$ and the number of ingested bacteria when compared with adult controls (MFI: 48.7, 33.9-69.2 versus 95, 52.2-138.2, $P<0.001)$. Furthermore, neonatal monocytes ingested significantly fewer bacteria than adult monocytes (MFI: 34.1, 25.3-45.1 versus 55.4, 41.6-68.2, $P<0.005$ ), and there was a trend to a smaller number of phagocytosing monocytes in neonates versus adults, but this did not reach statistical significance $(71.6 \%, 60.8-$ $75.9 \%$ versus $77.1 \%, 67.4-82.4 \% ; P=0.06$; Fig. 1 ).

Generation of oxidative burst products in fetal versus neonatal and adult neutrophils and monocytes. Significantly more unstimulated fetal granulocytes demonstrated synthesis of oxidative burst products in comparison with adult as well as neonatal granulocytes (percentage positive granulocytes: $4.1 \%$, $1.7-8 \%$ versus $0.8 \%, 0.3-1.7 \%, P<0.005$ and versus $0.8 \%$, $0.5-1.1 \% ; P<0.001$; Fig. $2 A)$. Stimulation with fMLP resulted in a significantly larger percentage of fetal and neonatal granulocytes positive for oxidative burst products in compari-

\section{Phagocytosis}

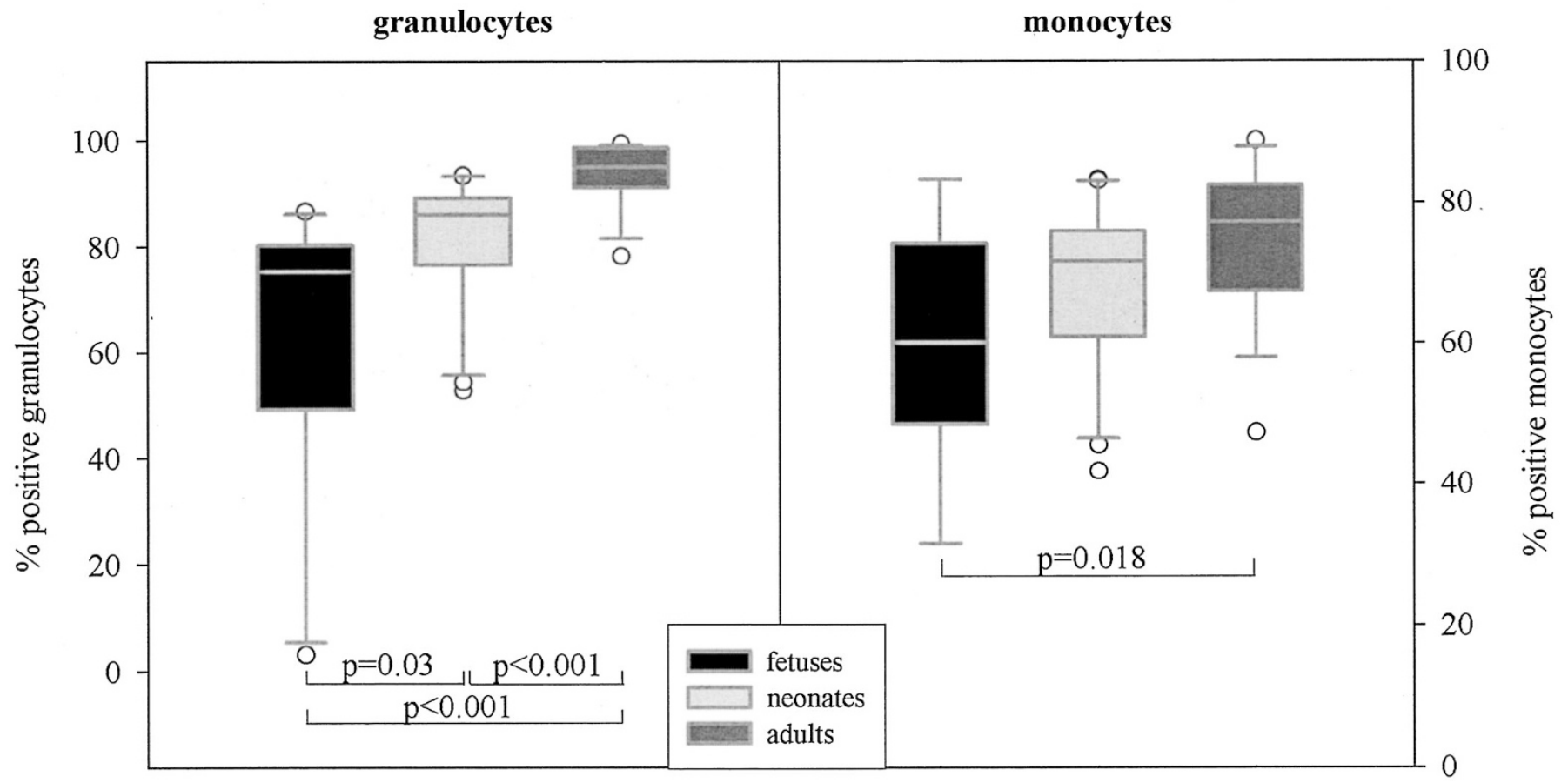

Figure 1. Phagocytosis of E. coli by fetal granulocytes and monocytes in comparison with neonates and adults. The percentage of fetal $(n=10)$, neonatal $(n=20)$, and adult $(n=15)$ granulocytes and monocytes positive for FITC-labeled $E$. coli is depicted. Phagocytosis was significantly impaired by fetal and neonatal granulocytes compared with adults. Furthermore, significantly fewer fetal monocytes ingested E. coli than did adult monocytes. Data were expressed as box-plots showing the 10th, 25th, 75th, and 90th percentiles. 
A

granulocyte respiratory burst

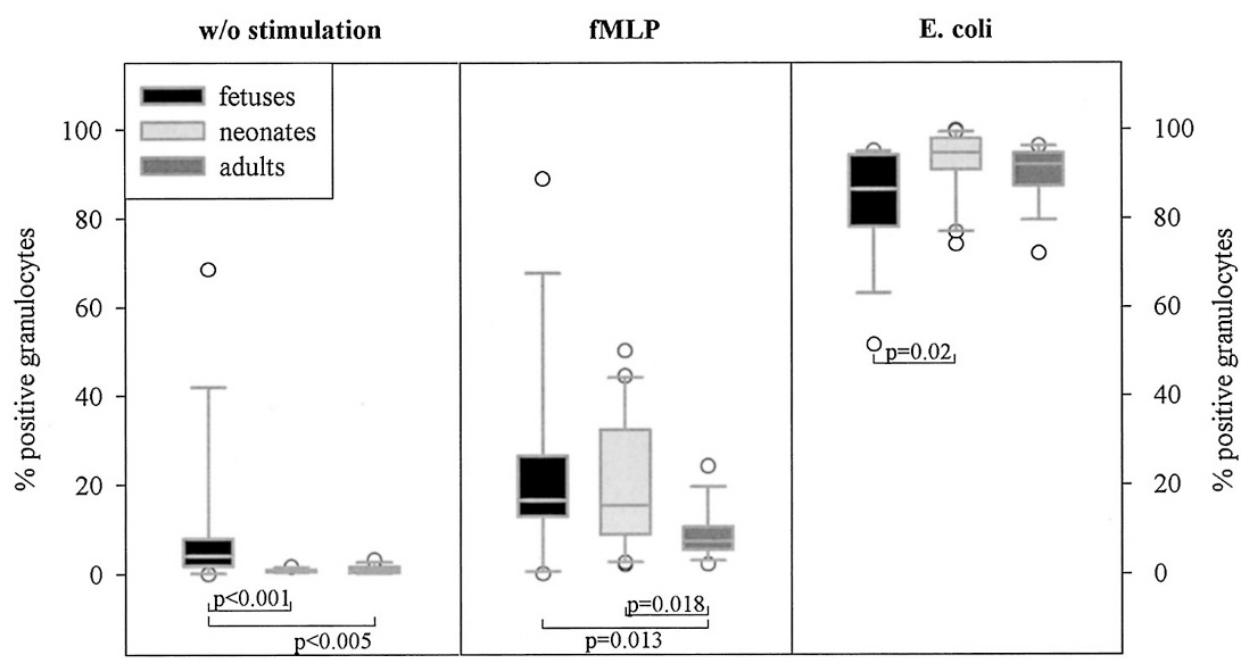

B monocyte respiratory burst

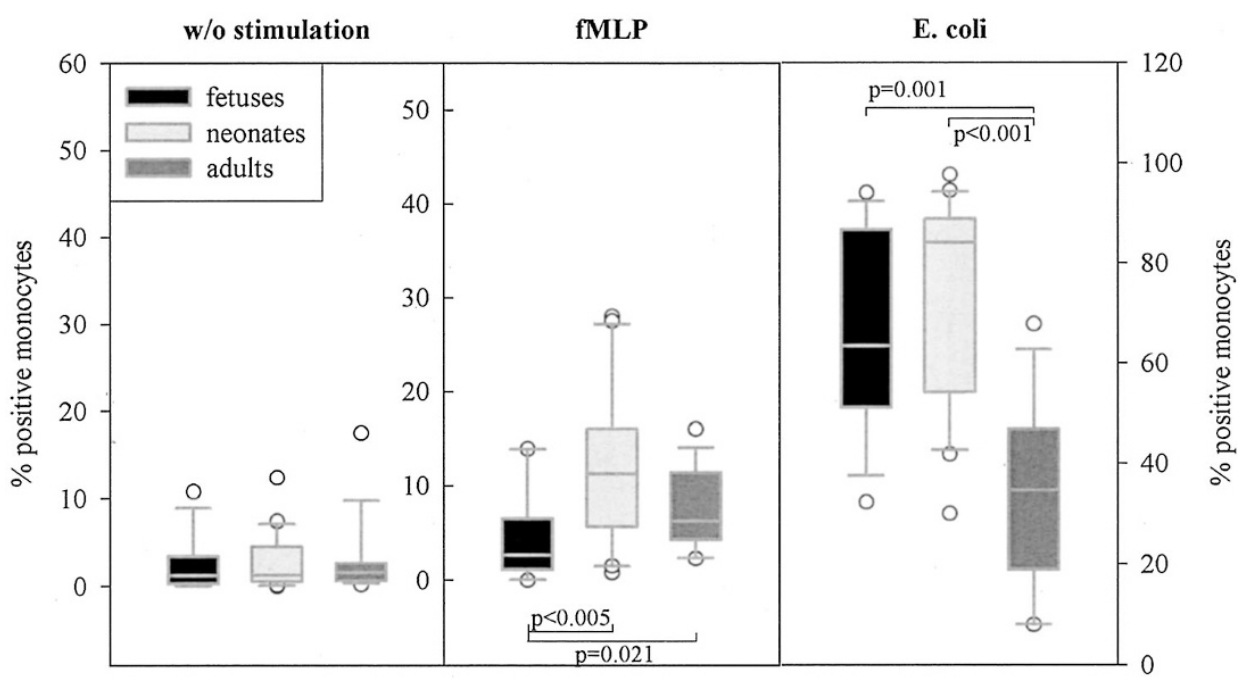

Figure 2. Synthesis of respiratory burst products in fetal granulocytes $(A)$ and monocytes $(B)$ in comparison with neonatal and adult leukocytes. The percentage of fetal $(n=14)$, neonatal $(n=20)$, and adult $(n=15)$ dihydrorhodamine-positive granulocytes $(A)$ and monocytes $(B)$ is shown. Data were expressed as box-plots showing the 10th, 25th, 75th, and 90th percentiles. Significantly more fetal granulocytes produced reactive oxygen products both spontaneously and after stimulation with fMLP than adults. After stimulation with fMLP, also more neonatal granulocytes produced burst products than adults. However, no differences were detected after stimulation with E. coli. Stimulation with fMLP resulted in a significantly lower number of monocytes positive for burst products in fetuses than in adults. Monocytes positive for burst products after stimulation with $E$. coli were significantly more frequent in both fetuses and neonates than in adults.

son with adults $(16.4 \%, 12.8-26.5 \%$ and $15.3 \%, 8.8-32.4 \%$, respectively, versus $7.4 \%, 5.5-10.5 \% ; P=0.013$ and $P=$ 0.018 ); however, percentages were comparable for fetuses and neonates. After stimulation with opsonized E. coli, number of positive granulocytes did not differ between fetuses and adults $(86.5 \%, 78-94.3 \%$ versus $92 \%, 87.3-94.7 \% ; P=0.2)$. However, neonates demonstrated a significantly higher number of oxidative burst product-positive granulocytes compared with fetuses $(94.6 \%, 90.1-98.0 \%$ versus $86.5 \%, 78.0-94.3 \%$; $P=$ $0.02)$ and a trend to higher values than adults $(92 \%, 87.3-$ $94.7 \% ; P=0.06)$.

With respect to the number of oxidative burst products, no differences in MFI were detected between the groups when unstimulated granulocytes were analyzed (fetuses: $3.6,2.8-$ 5.2; neonates: 3.4, 2.9-4.8; adults: 3.9, 1.9-7.6). However, fetal granulocytes displayed significantly lower MFI values after stimulation with fMLP, but a trend to higher MFI values after maximal stimulation with $E$. coli was found when compared with adults $[3.1,2.6-5.1$ versus $4.8,4.2-5.8(P=0.011)$ and $31.4,9.3-43.1$ versus $10.3,8-12.3(P=0.029)$, respectively). Neonatal granulocytes demonstrated comparable MFI values after application of fMLP but significantly higher MFI values after stimulation with $E$. coli in contrast to adults (28.2, $23.9-61.1$ versus $10.3,8.0-12.3 ; P<0.001)$.

Number of unstimulated monocytes positive for oxidative burst products were comparable among fetuses, neonates, and 
adults $(1.2 \%, 0.3-3.4 \%$ versus $1.3 \%, 0.6-4.5 \%$ versus $1.6 \%$, $0.6-2.6 \%$; Fig. 2B). However, fetuses displayed a lower number of positive monocytes after stimulation with fMLP compared with neonates and adults $(2.6 \%, 1.1-6.5 \%$ versus $11.3 \%$, $5.6-16.0 \%$ and $6.3 \%, 4.3-11.4 \% ; P<0.005$ and $P=0.021$, respectively). Fetuses and neonates showed a higher number of positive monocytes after stimulation with $E$. coli than adults $(63.4 \%, 51.2-86.7 \%$ and $84.1 \%, 54.1-88.8 \%$ versus $34.6 \%$, $18.9-46.8 \% ; P=0.001$ and $P<0.001$, respectively), whereas no differences between fetuses and neonates were detected $(P$ $=0.5$ ).

A direct negative correlation was observed between gestational age and oxidative burst products-positive monocytes both spontaneously and after stimulation with fMLP $(r=$ $-0.57, P<0.05$ and $r=-0.53, P<0.05$, respectively). A similar trend was found for fetal granulocytes; however, this did not reach statistical significance.

Fetal monocytes showed a trend to lower amounts of oxidative burst products in monocytes after stimulation with fMLP (MFI: 3.9, 2.7-7.9 versus 6.8, 4.9-11.6; $P=0.08$ ) but higher values after application of $E$. coli bacteria compared with adults (MFI: 25.7, 5.4-36 versus 6.3, 5.1-12.8; $P=$ 0.033 ). This result was similar to differences observed for fetal versus adult granulocytes.

Expression of adhesion molecules on fetal versus neonatal and adult granulocytes. Several adhesion molecules were expressed on a significantly lower number of fetal compared with neonatal and adult granulocytes. Frequency of CD11apositive cells was lower in fetuses than in neonates and adults $(45.7 \%, 12.9-66.6 \%$ versus $97.8 \%, 68.9-99.2 \%$ versus 97.3\%, 91.4-98.4\%; $P<0.005$ and $P<0.001$, respectively; Fig. 3). Similarly, expression of CD11b was detected on a smaller number of granulocytes in fetuses than the other groups $(63.3 \%, 47-79.8 \%$ versus $98.6 \%, 67.5-99.7 \%$ and $99.2 \%$,
96.4-99.7\%; $P<0.01$ and $P<0.001$, respectively; Fig. 3). Furthermore, the adhesion molecules CD18 (45.9\%, 26.1$74.1 \%$ versus $98.1 \%, 71.2-99.2 \%$ versus $93.5 \%, 87.1-98.3 \%$; $P<0.001$ and $P<0.001$, respectively; Fig. 3) and CD62L $(47.9 \%, 41-80.1 \%$ versus $97.5 \%, 92.9-99.1 \%$ versus $98.5 \%$, $96.8-99.7 \% ; P<0.001$ and $P<0.001$, respectively; Fig. 3) were found on a smaller number of fetal versus neonatal and adult granulocytes. A positive correlation was found for expression of CD62L and gestational age $(r=0.73, P<0.05)$. No significant differences were detected for the expression of CD11c on fetal, neonatal, and adult granulocytes (42.5\%, $10.5-67 \%$ versus $78.9 \%, 50.5-92.3 \%$ versus $70.5 \%, 25.8-$ $81 \% ; P=0.046$ and $P=0.2$, respectively; Fig. 3). MFI values were significantly lower for CD11b-positive granulocytes in fetuses versus neonates and adults $(5.7,3.5-10.4$ versus 9.1 , 7.5-16.2 versus $12.4,7.6-19 ; P=0.02$ and $P<0.01$, respectively). However, no significant differences were detected for MFI values of the other surface markers under investigation.

Production of proinflammatory cytokines by monocytes in fetuses compared with healthy adults. After stimulation with lipopolysaccharide, a significantly smaller number of fetal monocytes were detected positive for IL-6 (41.7\%, 32.7$47.0 \%$ versus $60.2 \%, 48.7-66.5 \% ; P<0.005$ ) as well as for TNF- $\alpha$ compared with healthy adult controls $(15.8 \%, 12.9-$ $17.6 \%$ versus $29.4 \%, 21.1-45.8 \% ; P<0.001)$. However, significantly more fetal monocytes displayed positivity for IL-8 $(87.8 \%, 69.8-91.6 \%$ versus $74 \%, 62.0-80.8 \%$; $P<0.01$; Fig. 4). A positive correlation was observed between the number of IL-8 -producing monocytes and gestational age $(r=0.69, P<$ 0.05 ). When compared with healthy neonates [primary data published in 24)], fetuses showed a significantly impaired synthesis of IL-6 and TNF- $\alpha$ but comparable number of IL8 -positive monocytes.

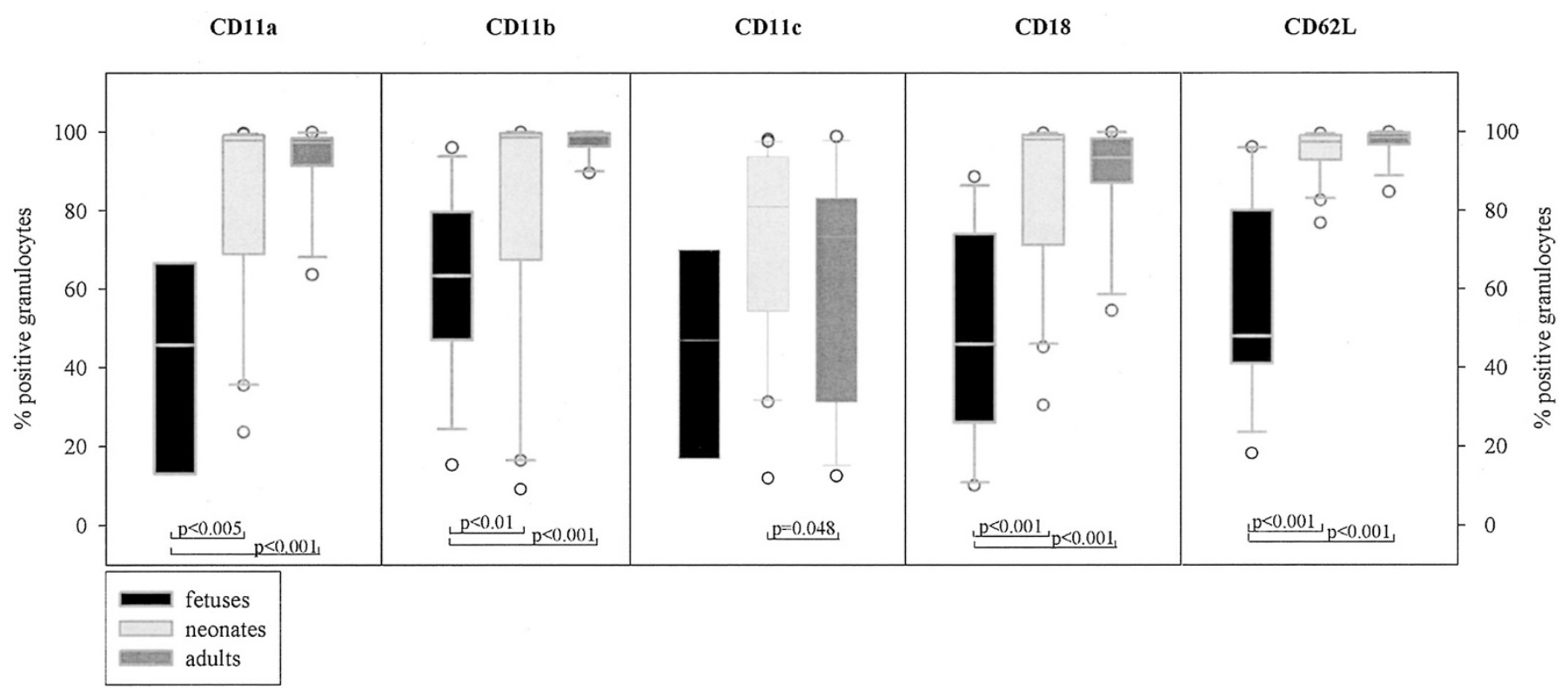

Figure 3. Expression of surface markers on fetal $v s$ neonatal and adult granulocytes. The percentage of granulocyte-positive adhesion molecules is depicted. Data were expressed as box-plots showing the 10th, 25th, 75th, and 90th percentiles. The frequencies of fetal granulocytes positive for CD11a $(n=7)$, CD11b $(n=14), \operatorname{CD} 18(n=13)$, and CD62L $(n=14)$ were significantly lower in fetuses than in neonates $(n=20)$ and adults $(n=15)$, whereas the number of CD11c-positive granulocytes $(n=7)$ did not differ significantly between groups. 


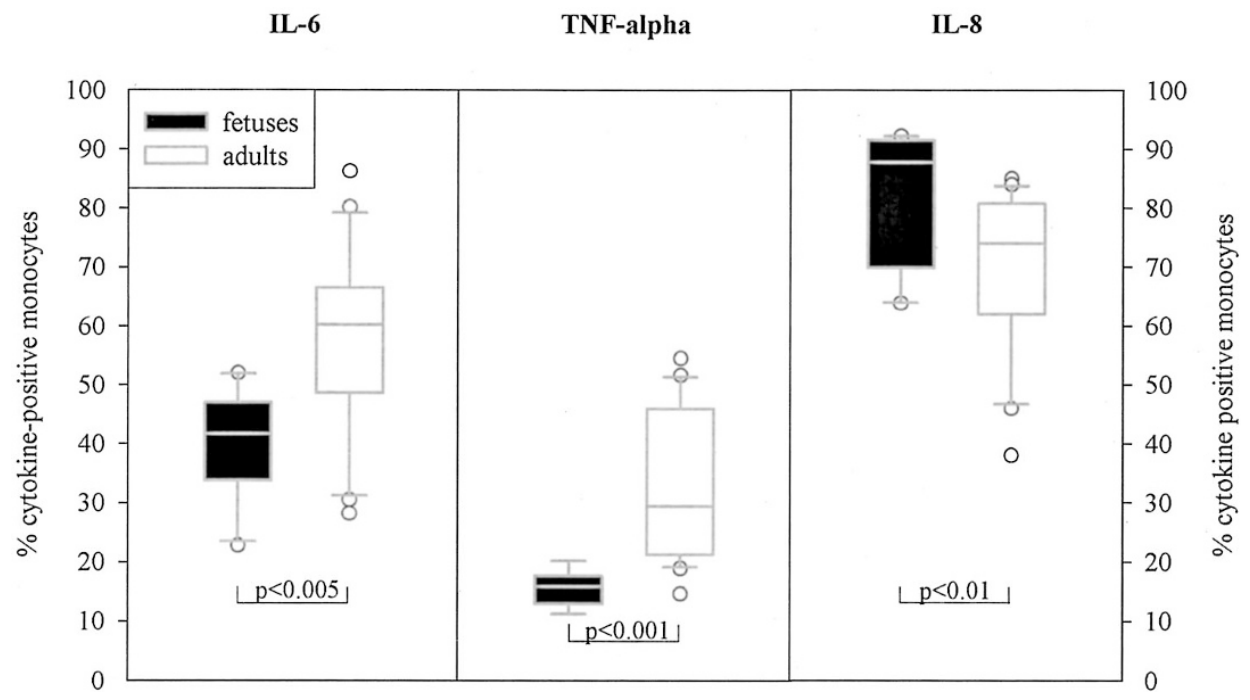

Figure 4. Synthesis of IL-6, TNF- $\alpha$, and IL- 8 in fetal and adult monocytes. Cytokine production after endotoxin challenge with lipopolysaccharide. Data were expressed as box-plots showing the 10th, 25th, 75th, and 90th percentiles. The percentage of fetal monocytes positive for IL-6 $(n=10)$ and TNF- $\alpha(n=9)$ was lower than in adults $(n=15)$. However, significantly more fetal $(n=12)$ than adult monocytes were detected positive for IL-8.

\section{DISCUSSION}

Only a few data are available to date describing innate immune responses in human fetuses before birth. In our study, we used flow cytometry to determine the expression of adhesion molecules, phagocytic activity, and oxidative burst as well as the synthesis of proinflammatory cytokines at the single-cell level. Flow cytometry allows for highly specific and valid measurement of these parameters from small blood samples that are available from human fetuses. In addition, the use of whole blood samples avoids artifactual changes in function induced by purification procedures (25). To eliminate the influence of stress factors such as labor $(26,27)$ or infection and to assess the maturation of the phagocyte immune response, we investigated neutrophils and monocytes obtained from human fetuses by percutaneous intrauterine umbilical vein sampling and compared it with cord blood from healthy neonates as well as peripheral blood from adults. We are aware that the fetuses were eligible for research investigations only because cord blood sampling was indicated for medical reasons, i.e. abnormalities detected in routine investigations during pregnancy. However, in our opinion, it would not be ethically justifiable to take cord blood samples from healthy, normal fetuses. To our best knowledge, we do not think that the abnormalities detected are essentially related to significant dysfunction of the developing innate immune system that would prevent careful interpretation of our data.

Several investigators demonstrated normal expression of CD11b (CR3) on polymorphonuclear cells of term neonates; however, preterm infants were shown to have a diminished expression of this marker (28-30). Furthermore, a failure to appropriately up-regulate $\mathrm{CD} 11 \mathrm{~b}$ in response to stimuli $(20,28,30)$ could be demonstrated to be due to a decreased total cellular content of $\mathrm{CD} 11 \mathrm{~b}$, correlating with gestational age (18). In contrast, Rebuck et al. (31) suggested that these results are due to differential sensitivity of leukocytes to isolation techniques. In our study, a diminished surface expression of
CD11b on whole-blood fetal granulocytes was shown. The most likely explanation for this observation is that in a more immature organism such as fetuses, an even lower total cellular content of $\mathrm{CD} 11 \mathrm{~b}$ translates into decreased surface expression. In addition, we found a significantly decreased expression of CD11a and CD18 in fetuses but a normal number of positive granulocytes in term neonates compared with adults. These results are in accordance with earlier data describing an essentially impaired expression of these adhesion molecules in preterm infants but normal values for mature newborns (18). This physiologic immaturity of neutrophil adhesion molecule expression certainly adds to the impaired innate immune response observed in preterm neonates. The importance of these $\beta_{2}$-integrins for the innate host defense are illustrated by the leukocyte adhesion defect type I, whereby the near absence of CD11a/CD18 results in significant immunodeficiency (32).

CD62L (L-selectin) is a leukocyte adhesion molecule that is important in the early stage of the interaction of leukocytes with vascular endothelium during the process of emigration from the bloodstream (33). In this study, a significantly decreased expression of CD62L on fresh whole-blood granulocytes was detected compared with healthy neonates and adults, whereas values of the last two groups did not differ significantly. Furthermore, a direct positive correlation between gestational age and number of CD62L-positive fetal granulocytes was found. This is in keeping with data obtained from umbilical cord CD34 hematopoietic progenitors (34), indicating a maturation of adhesion molecule expression over time. However, normal levels of CD62L in fetuses were depicted by Smith et al. (21), and both positive and negative correlation of soluble CD62L with gestational age were described by Rebuck et al. (31) and Buhrer et al. (35). Several other groups reported lower expression of CD62L on granulocytes and monocytes in term and preterm infants than in adults $(19,20,36,37)$. The differences observed between researchers may be due to differences in setup of assays, i.e. usage of whole blood or 
purification procedures as well as storage time until analysis. As CD62L was shown to be significantly down-regulated in response to various stimuli $(37,38)$, in our opinion, data obtained from fresh whole-blood granulocytes are most reliable.

Phagocytosis by granulocytes and monocytes is one of the early steps involved in antibacterial innate host defense, impairment of which can result in significant immune deficiency. The majority of the currently available data indicate that most preterm infants, clinically well or stressed, have an impaired phagocytic activity (39). However, some investigators have observed normal phagocytic activity even in stressed neonates (7). In our analyses, the phagocytic activity of fetal neutrophils and monocytes was significantly diminished compared with healthy neonates and adults. Furthermore, a direct positive relationship between gestational age and number of phagocytosing granulocytes could be demonstrated. Neonates displayed a higher number of phagocytes positive for bacteria and greater amounts of ingested E. coli than fetuses, but both levels were smaller again compared with adults. These results are in keeping with studies demonstrating decreased neutrophil phagocytic activity in preterm infants in comparison with term neonates and adults $(17,39)$, and taken together, our findings indicate a gradual maturation of phagocytic capacity over time. As fully opsonized E. coli bacteria were used for the assessment of phagocytosis, possible lack of opsonizing proteins in fetal serum, mainly complement and immunoglobulins, is unlikely to underlie the differences observed. Taking into account the diminished expression of $\mathrm{CD} 11 \mathrm{~b}$ on fetal neutrophils shown in our study, we speculate that the decreased expression of one of the most important surface receptors involved in phagocytosis (3) contributes to impaired phagocytosis in fetuses. However, neither Falconer et al. (38) nor we were able to detect a direct correlation between phagocytosis and expression of CD11b, which might be due to the limited number of individuals investigated in both studies.

The neutrophil respiratory burst activity (NRBA) is essential for intracellular killing of phagocytosed bacteria. Thus, an impairment of the NRBA results in significant impairment of the host immune defense with possible fatal consequences as obvious in chronic granulomatous disease (39). The ability of neonatal phagocytes to produce bactericidal oxygen metabolites has been studied previously by several investigators; however, results were conflicting. Most of the groups demonstrated normal or even enhanced NRBA by neutrophils of term and preterm infants $(9,17,39-41)$, and a positive correlation to gestational age was observed (2). In contrast, other researchers depicted normal NRBA in healthy term neonates but diminished NRBA in both preterm infants and infected or stressed neonates $(7,8,11,12)$. In our study, we excluded the influence of labor on the phagocyte function by analyzing fetal blood $(25,26)$. To our knowledge, only Newburger $(42)$ investigated the ability of fetal neutrophils to produce oxidative burst products and demonstrated comparable results for fetuses and adults. Here, we could demonstrate an even higher percentage of activated phagocytes in fetuses compared with healthy adults both spontaneously and after stimulation with fMLP. Thus, these findings are in keeping with Newburger's (42), indicating the early maturation of the NADPH oxidase system as a first-line defense mechanism of innate immunity. Furthermore, we observed a direct negative correlation of the oxidative burst product generation to gestational age. The enhanced oxidative burst activity observed thus might be interpreted as an intrinsic mechanism to overcome the immaturity of the phagocytic capacity. This, however, could also have some detrimental side effects as an exaggerated oxygen radical formation may be involved in some diseases characteristic of preterm infants, e.g. bronchopulmonary dysplasia and retinopathy of prematurity (43).

In the current study, we could demonstrate that fetal monocytes are capable of significant synthesis of IL-6, IL-8, and TNF- $\alpha$. However, the number of IL- $6-$ and TNF- $\alpha$-positive monocytes was significantly smaller than in neonates and adults. In contrast, significantly more fetal than adult monocytes produced IL-8, and the number of IL-8-positive monocytes was positively correlated with gestational age. These data are well in keeping with our previous results (24) that showed enhanced synthesis of IL- 6 and IL-8 in term and preterm infants ( $>32$ wk of gestation) compared with healthy adults. Contrastingly, very preterm infants $(<32 \mathrm{wk})$ displayed diminished number of IL-6-positive monocytes. However, even very preterm infants were able to mount significant IL-6 production when primed by in vivo infection. No difference in number of TNF- $\alpha$-positive monocytes was detected between term and preterm infants and healthy adults. These results might indicate that IL-8 production matures very early during gestation, whereas a comparable number of IL-6- and TNF$\alpha$-positive monocytes are found after 32 wk of gestation or in response to intensive in vivo stimulation. Thus, it may be speculated that the production of proinflammatory cytokines could be more essentially involved in the pathogenesis of several neonatal diseases than previously thought, even in very immature infants (44).

In conclusion, in the present study, it could be shown that the fetal innate immune response is essentially different from that observed in term neonates and adults. Fetal phagocytes display a differential maturation of several inflammatory functions: a significantly diminished phagocytic capacity was demonstrated, whereas the generation of the respiratory burst products was enhanced. Furthermore, expression of several adhesion molecules essentially involved in adhesion, migration, and phagocytosis was also found to be significantly decreased. Fetal monocytes demonstrate the capacity to produce proinflammatory cytokines in response to stimulation; however, the pattern of production is different from more mature individuals. The results of our study add significantly to our understanding of the maturation of the innate immune response.

\section{REFERENCES}

1. Gladstone IM, Ehrenkranz RA, Edberg SC, Baltimore RS 1990 A ten-year review of neonatal sepsis and comparison with the previous fifty-year experience. Pediatr Infect Dis J 9:819-825

2. Gessler P, Luders R, Konig S, Haas N, Lasch P, Kaschel W 1995 Neonatal neutropenia in low birthweight premature infants. Am J Perinatol 12:34-38

3. Carr R 2000 Neutrophil production and function in newborn infants. Br J Hematol 110:18-28

4. Stoll BJ, Gordon T, Korones SB, Shankaran D, Tyson JE, Bauer CR, Fanaroff AA, Lemons JA, Donovan EF, Oh W, Stevenson DK, Ehrenkranz RA, Papile LA, Verter J, Wright LL 1996 Late-onset sepsis in very low birthweight neonates: a report from 
the National Institute of Child Health and Human Development Neonatal Research Network. J Pediatr 129:63-71

5. Mease AD 1990 Tissue neutropenia: the newborn neutrophil in perspective. J Perinatol 10:55-59

6. Ohls RK, Li Y, Abdel-Mageed A, Buchanan G Jr, Mandell L, Christensen RD 1995 Neutrophil pool sizes and granulocyte colony-stimulating factor production in human mid-trimester fetuses. Pediatr Res 37:806-811

7. Shigeoka AO, Charette RP, Wyman ML, Hill HR 1981 Defective oxidative metabolic responses of neutrophils from stressed neonates. J Pediatr 98:392-398

8. Peden DB, VanDyke K, Ardekani A, Mullett MD, Myerberg DZ, VanDyke C 1987 Diminished chemiluminescent responses of polymorphonuclear leukocytes in severely and moderately preterm neonates. J Pediatr 111:904-906

9. Bektas S, Goetze B, Speer CP 1990 Decreased adherence, chemotaxis and phagocytic activities of neutrophils from preterm neonates. Acta Paediatr Scand 79:1031-1038

10. Driscoll MS, Thomas VL, Ramamurthy RS, Casto DT 1990 Longitudinal evaluation of polymorphonuclear leukocyte chemiluminescence in premature infants. J Pediatr 116:429-434

11. Usmani SS, Schlessel JS, Sia CG, Kamran S, Orner SD 1991 Polymorphonuclear leukocyte function in the preterm neonate: effect of chronologic age. Pediatrics $87: 675-679$

12. Drossou V, Kanakoudi F, Tzimouli V, Sarafidis K, Taparkou A, Bougiouklis D, Petropoulou T, Kremenopoulos G 1997 Impact of prematurity, stress and sepsis on the neutrophil respiratory burst activity of neonates. Biol Neonate 72:201-209

13. Källman J, Schollin J, Schalèn C, Erlandsson A, Kihlström E 1998 Impaired phagocytosis and opsonisation towards group B streptococci in preterm neonates. Arch Dis Child Fetal Neonatal Ed 78:F46-F50

14. Levy O, Martin S, Eichenwald E, Ganz T, Valore E, Carroll SF, Lee K, Goldmann D, Thorne GM 1999 Impaired innate immunity in the newborn: newborn neutrophils are deficient in bactericidal/permeability-increasing protein. Pediatrics 104:1327-1333

15. Nupponen I, Turunen R, Nevalainen T, Peuravuori H, Pohjavuori M, Repo H, Andersson S 2002 Extracellular release of bactericidal/permeability-increasing protein in newborn infants. Pediatr Res 51:670-674

16. Mills EL, Thompson T, Björksten B, Filipovich D, Quie PG 1979 The chemiluminescence response and bactericidal activity of polymorphonuclear neutrophils from newborns and their mothers. Pediatrics 63:429-434

17. Habermehl P, Hauer T, Mannhardt W, Knuf M, Zepp F, Schofer O 1999 Granulocyte function in premature infants before the 34th week of pregnancy and in mature newborn infants. Klin Padiatr 211:149-153

18. McEvoy LT, Zakem-Cloud H, Tosi MF 1996 Total cell content of CR3 (CD11b/ CD18) and LFA-1 (CD11a/CD18) in neonatal neutrophils: relationship to gestational age. Blood 87:3929-3933

19. Murphy FJ, Reen DJ 1996 Differential expression of function-related antigens on newborn and adult monocyte subpopulations. Immunology 89:587-591

20. Kim SK, Keeney SE, Alpard SK, Schmalstieg FC 2002 Comparison of L-selectin and $\mathrm{CD} 11 \mathrm{~b}$ on neutrophils of adults and neonates during the first month of life. Pediatr Res 53:132-136

21. Smith JB, Tabsh KM 1993 Fetal neutrophils and eosinophils express normal levels of L-selectin. Pediatr Res 34:253-257

22. Schultz C 2002 Intracytoplasmic detection of proinflammatory cytokines and chemokines in monocytes by flow cytometry. In: Körholz D, Kiess W (eds) Cytokines and Colony Stimulating Factors, Methods and Protocols-Part II: Detection Assays for Cytokines and Growth Factors. Methods in Molecular Biology. Humana Press, Totowa, pp 29-39

23. Schultz C, Rott C, Temming P, von Puttkammer J, Bucsky P 2002 Influence of specimen age and use of different negative controls in determination of intracytoplasmic levels of cytokine after whole-blood culture assay. Clin Diagn Lab Immunol 9:295-298
24. Schultz C, Rott C, Temming P, Schlenke P, Möller JC, Bucsky P 2002 Enhanced interleukin-6 and interleukin- 8 synthesis in term and preterm infants. Pediatr Res 51:317-322

25. De Groote D, Zangerle PF, Gevaert Y, Fasotte MF, Beguin Y, Noizat-Pirenne F, Pirenne J, Gathy R, Lopez M, Dehart I, Igot D, Baufrihaye M, Delacroix, Franchimont P 1992 Direct stimulation of cytokines (IL-1 $\beta$, TNF- $\alpha$, IL-6, IL-2, IFN- $\gamma$, and GM-CSF) in whole blood. I. Comparison with isolated PBMC stimulation. Cytokine $4: 239-248$

26. Kinoshita Y, Masuda K, Kobayashi Y 1991 Adherence of cord blood neutrophils: effect of mode of delivery. J Pediatr 118:115-117

27. Gessler P, Dahinden C 2003 Increased respiratory burst and increased expression of complement receptor-3 (CD11b/CD18) and of IL-8 receptor-A in neutrophil granulocytes from newborns after vaginal delivery. Biol Neonate 83:107-112

28. Bruce MC, Baley JE, Medvik KA, Berger M 1987 Impaired surface membrane expression of $\mathrm{C} 3 \mathrm{bi}$ but not $\mathrm{C} 3 \mathrm{~b}$ receptors on neonatal neutrophils. Pediatr Res 21:306-311

29. Falconer AE, Carr R, Edwards SW 1995 Neutrophils from preterm neonates and adults show similar cell surface receptor expression: analysis using a whole blood assay. Biol Neonate 67:26-33

30. Smith JB, Kunjummen RD, Raghavender BH 1991 Eosinophils and neutrophils of human neonates have similar impairments of quantitative up-regulation of Mac-1 (CD11b/CD18) expression in vitro. Pediatr Res 30:355-361

31. Rebuck N, Gibson A, Finn A 1995 Neutrophil adhesion molecules in term and premature infants: normal or enhanced leukocyte integrins but defective L-selectin expression and shedding. Clin Exp Immunol 101:183-189

32. Inwald D, Davies EG, Klein N 2001 Demystified: adhesion molecule deficiencies. Mol Pathol 54:1-7

33. Bevilacqua MP, Nelson RM 1993 Selectins. J Clin Invest 91:379-387

34. Surbek DV, Steinmann C, Burk M, Hahn S, Tichelli A, Holzgreve W 2000 Developmental changes in adhesion molecule expressions in umbilical cord blood CD34 hematopoietic progenitor and stem cells. Am J Obstet Gynecol 183:1152-1157

35. Buhrer C, Stibenz D, Graulich J, Gernhold U, Butcher EC, Dudenhausen JW, Obladen M 1995 Soluble L-selectin (sCD62L) umbilical cord plasma levels increase with gestational age. Pediatr Res 38:336-341

36. Torok C, Lundahl J, Hed J, Lagercrantz H 1993 Diversity in regulation of adhesion molecules (Mac-1 and L-selectin) in monocytes and neutrophils from neonates and adults. Arch Dis Child 68:561-565

37. Buhrer C, Graulich J, Stibenz D, Dudenhausen JW, Obladen M 1994 L-selectin is down-regulated in umbilical cord blood granulocytes and monocytes of newborn infants with acute bacterial infection. Pediatr Res 36:799-804

38. Falconer AE, Carr R, Ewards SW 1995 Impaired neutrophil phagocytosis in preterm neonates: lack of correlation with expression of immunoglobulin or complement receptors. Biol Neonate 68:264-269

39. Boxer LA 2000 Disorders of phagocyte function. In: Behrman RE, Kliegman RM, Jenson HB (eds) Nelson Textbook of Pediatrics. WB Saunders Company, Philadelphia, pp 619-621

40. Ambruso DR, Altenburger KM, Johnston RB Jr 1979 Defective oxidative metabolism in newborn neutrophils: discrepancy between superoxide anion and hydroxyl radical generation. Pediatrics 64:722-725

41. Yamazaki M, Matsuoka T, Yasui K, Komiyama, Akabane T 1988 Increased production of superoxide anion by neonatal polymorphonuclear leukocytes stimulated with a chemotactic peptide. Am J Hematol 27:169-173

42. Newburger PE 1982 Superoxide generation by human fetal granulocytes. Pediatr Res $16: 373-376$

43. Weiss SJ 1989 Tissue destruction by neutrophils. N Engl J Med 320:365-376

44. Gomez R, Romero R, Ghezzi F, Yoon BH, Mazor M, Berry SM 1998 The fetal inflammatory response syndrome. Am J Obstet Gynecol 179:194-202 